\title{
Synthesis of Advanced Electrokinetic Technologies in Agroecosystems
}

\author{
E.G. Porsev \\ Novosibirsk State Technical University \\ Novosibirsk, Russian Federation \\ e-mail: porseve@mail.ru
}

\author{
B.V. Malozyomov \\ Novosibirsk State Technical University \\ Novosibirsk, Russian Federation \\ e-mail: mbv5@mail.ru
}

\begin{abstract}
It was proposed to use electrokinetic phenomena for the cultivation of agricultural products, taking into account the multifactority of technology and the presence of relaxation phenomena. It is forecasted to use new approaches to reduce energy costs for the process of moistening the root-layer of soil and saving natural resources. The proposed technology will make it possible to get rid of a number of irrationally used natural resources. This is especially true for the Crimean region and the Volga region. Using this technology in Uzbekistan will allow the elimination of the Aral catastrophe and restoration of the natural balance in this region.
\end{abstract}

Keywords- electical discharge, electricity, current, voltage, electrotechnology, electroosmosis

\section{INTRODUCTION}

Electroosmotic flow (or electro-osmotic flow, often abbreviated EOF; synonymous with electroosmosis or electroendosmosis) is the motion of liquid induced by an applied potential across a porous material, capillary tube, membrane, microchannel, or any other fluid conduit. Because electroosmotic velocities are independent of conduit size, as long as the electrical double layer is much smaller than the characteristic length scale of the channel, electroosmotic flow will have little effect. Electroosmotic flow is most significant when in small channels. Electroosmotic flow is an essential component in chemical separation techniques, notably capillary electrophoresis. Electroosmotic flow can occur in natural unfiltered water, as well as buffered solutions.

Electroosmotic flow was first reported in 1809 by F. F. Reuss in the Proceedings of the Imperial Society of Naturalists of Moscow. He showed that water could be made to flow through a plug of clay by applying an electric voltage. Clay is composed of closely packed particles of silica and other minerals, and water flows through the narrow spaces between these particles just as it would through a narrow glass tube. Any combination of an electrolyte (fluid containing dissolved ions) and an insulating solid would generate electro-osmotic flow, though for water/silica the effect is particularly large. Even so, flow speeds are typically only a few millimeters per second

Electroosmotic flow is caused by the Coulomb force induced by an electric field on net mobile electric charge in a solution. Because the chemical equilibrium between a solid surface and an electrolyte solution typically leads to the interface acquiring a net fixed electrical charge, a layer of mobile ions, known as an electrical double layer or Debye layer, forms in the region near the interface. When an electric field is applied to the fluid (usually via electrodes placed at inlets and outlets), the net charge in the electrical double layer is induced to move by the resulting Coulomb force. The resulting flow is termed electroosmotic flow.

The resulting flow from applying a voltage is a plug flow. Unlike a parabolic profile flow generated from a pressure differential, a plug flow's velocity profile is approximately planar, with slight variation near the electric double layer. This offers significantly less deleterious dispersive effects and can be controlled without valves, offering a high performance method for fluid separation, although many complex factors prove this control difficult. Because of difficulties measuring and monitoring flow in micro fluidic channels, primarily disrupting the flow pattern, most analysis is done through numerical methods and simulation [1]. Electroosmotic flow through micro channels can be modeled after the NavierStokes equation with the driving force deriving from the electric field and not the pressure differential.

Electro-osmotic flow is commonly used in microfluidic devices [2, 3], soil analysis, processing [4], and chemical analysis [5], all of which routinely involve systems with highly charged surfaces, often of oxides. One example is capillary electrophoresis in which electric fields are used to separate chemicals according to their electrophoretic mobility by applying an electric field to a narrow capillary, usually made of silica. In electrophoretic separations, the electroosmotic flow affects the elution time of the analytes.

Electroosmotic flow is actuated in a FlowFET to electronically control fluid flow through a junction.

It is projected that micro fluidic devices utilizing electroosmotic flow will have applications in medical research. Once controlling, this flow is better understood and implemented; the ability to separate fluids on the atomic level will be a vital component for drug dischargers. Mixing fluids at the micro scale is currently troublesome. It is believed that electrically controlling fluids will be the method in which small fluids are mixed. 
A controversial use of electro-osmotic systems is being done to control rising damp in the structure of a building. While there is little evidence to suggest that these systems can be useful in moving salts in walls, such systems are claimed to be especially effective in structures with very thick walls. However, some claim that there is no scientific base for those systems and cite several examples for their failure.

In vascular plant biology, electro-osmosis is also used as an alternative or supplemental explanation for the movement of polar liquids via the phloem that differs from the cohesiontension theory supplied in the mass flow hypothesis and others, such as cytoplasmic streaming. Companion cells are involved in the "cyclic" withdrawal of ions $(\mathrm{K}+)$ from sieve tubes, and their secretion parallel to their position of withdrawal between sieve plates, resulting in polarisation of sieve plate elements alongside potential difference in pressure, and results in polar water molecules and other solutes present moved upward through the phloem.

In 2003, St Petersburg University graduates applied direct electric current to $10 \mathrm{~mm}$ segments of mesocotyls of maize seedlings alongside one-year linden shoots; electrolyte solutions present in the tissues moved toward the cathode that was in place, suggesting that electro-osmosis might play a role in solution transport through conductive plant tissues.

Maintaining an electric field in an electrolyte requires Faradaic reactions to occur at the anode and cathode. This is typically electrolysis of water, which generates hydrogen peroxide, hydrogen ions (acid) and hydroxide (base) as well as oxygen and hydrogen gas bubbles. The hydrogen peroxide and/or $\mathrm{pH}$ changes generated can adversely affect biological cells and biomolecules such as proteins, while gas bubbles tend to "clog" microfluidic systems. These problems can be alleviated by using alternative electrode materials such as conjugated polymers which can undergo the Faradaic reactions themselves, dramatically reducing electrolysis.

The advanced crop cultivation technology in agriculture alternative to traditional is using cultivation of crops with the use of electrowatering. It was first put forward by H. Opitz.

Traditional technology involves the construction of a system of irrigation canals and irrigation of crops by sprinkling machine.

At the same time, part of the effective area is occupied by irrigation canals and service lanes for sprinkling equipment, i.e. is actually not used for the purpose of phytoproduction and besides, a number of undesirable phenomena arise: in the upper wet soil layer fungi, mosses develop; slugs grow; intensive fertilization removes fertilizers from the soil; pollutes subsoil waters, slackens phytoproductivity.

A new method of moistening the root layer is to use the phenomenon of electroosmosis. In the root layer of soil (25-30 $\mathrm{cm}$ ), a system of conductors is placed and connected to the negative pole of the DC source. At the groundwater level, a second electrode is installed - a steel pipe connected to the positive pole of the source.
Electrodes are supplied with electrical voltage from 2 to 12 $V$ depending on the structure of the soil and the content of salts in it. Under the influence of an electric field, ground waters are pulled upwards and constantly moisten the roots of plants. At the same time, water is purified from salts deposited at the anodes, which is important for brackish soils.

The surface of the soil remains dry and friable, unwanted organisms are not found in it. Besides, due to electrodialysis, the moisture supplied to the plants acquires an alkaline reaction, which provides stimulation of plant growth.

Electrokinetic phenomena reflect the relationship that exists between the relative motion of two phases and the electrical properties of their boundary line.

Electrokinetic phenomena arise when one phase is dispersed into another, that is, when the system can be characterized as microheterogeneous. There are four groups of electrokinetic phenomena according to Antropov [1]: electroosmosis, electrophoresis, flow potential and deposition potential.

Taking into account the tradition in physics, the flow potential, in our opinion, is called the potential difference or the 'osmo' electromotive force (osmo-emf), and the deposition potential is the 'foro' electromotive force (foro-emf). These terms were offered by the authors firstly.

So, according to modern concepts, the process of electrokinetic energomass transfer is a mass transfer in colloid systems when an external electric field is applied or accompanied by the production of the own electric field in a colloidal system.

Colloidal systems consist of two phases: a dispersed phase and a dispersion medium, that is, they are heterogeneous. The work spent on the fragmentation of the substance to break the bonds between the molecules accumulates in the form of the potential energy of unsaturated bonds at the phase interface. With a colossal surface area, this excess surface energy reaches large values.

Thus, a substance in a colloidal state has a higher energy, greater activity than an undigested substance of the same composition.

In this case, the main cause of the phenomenon of electrokinetic energy and mass transfer is the existence of a double electric layer (DEL): in an electrically conductive dispersive medium around the dispersed particle of a dielectric material, adsorption of ions of predominantly one sign uncharged surfaces occurs due to the dispersive forces that result from the fluctuation of the electron shells of atoms. These forces are called the forces of specific adsorption.

The latter depends on the polarization, i.e. the effect of a given ion on atoms on the surface, and the ability to deform their electronic shells. The polarizing force of the ion depends on the strength of its electric field. 


\section{THEORY}

According to the classical theory of Gui-Chapman, the model of a double electric layer is based on the idea of the mobility of ions in the outer shell.

The electrostatic attraction of them to the surface and the repulsion of ions charged with the same name with the surface is balanced by thermal motion of ions, which erodes surface excesses.

The given state equilibrium distribution forms a "cloud" of electric charges near the surface of the solution with decreasing density, analogous to the distribution of the density of gases in the atmosphere.

In the electric field, counterions of the diffuse layer of DEL, energetically weakly bound to the surface of the solid phase (membrane), will move to the corresponding electrode and due to molecular friction, entrain the dispersion medium (aqueous solution of electrolytes).

The greater the electric field strength and the thickness of the diffuse layer, the greater the charge carriers, the faster the fluid is transported in the porous body or the dispersed particle in the electrically conductive dispersion medium.

In this case, the volumetric velocity of the liquid during electroosmosis is described by the equation:

$$
V=\frac{I \cdot \varepsilon \cdot \varepsilon_{0} \cdot \xi}{4 \cdot \pi \cdot \eta \cdot \gamma}
$$

where $I$ - the strength of current; $\varepsilon$ - the dielectric constant of the liquid; $\varepsilon_{0}$ - the dielectric constant of the vacuum; $\boldsymbol{\xi}$ - electrokinetic potential; $\eta$ - coefficient of internal friction (viscosity); $\gamma$ - specific conductivity of the liquid.

Equation 1, called the Helmholtz-Smoluchowski equation, is often written relative to the electrokinetic potential:

$$
\xi=\frac{\eta \cdot U_{0}}{\varepsilon \cdot \varepsilon_{0} \cdot E},
$$

where $U_{0}$ - the constant linear velocity of the liquid through the membrane, $E$ - the strength of the electric field.

In the case of a homeoporous membrane, that is, consisting of capillaries of the same cross-section, with an increase in the radius of the capillaries, while maintaining a constant gradient of the potential of the external field on the membrane, it is necessary to achieve a ratio in which a steady flow of liquid in the capillaries can not occur due to large inertia forces.

At the same time, $V, I$ and $\xi$ should turn to "0". A homeoporous membrane sometimes occurs with electroosmosis in clays, sandy loam and so on, where the homogeneity of the dispersed fraction is relatively large, that is, it is most often found in construction and chemical industries.

However, the real membrane characteristic of agroproduction is a heteroporous membrane that is containing capillaries of different radii and characterized by a certain pore size distribution curve.

An increase in the pore radius of such membrane should lead to the fact that in the largest capillaries at a given potential gradient, the driving electric force, equal to the product $\operatorname{grad} \varphi$ of the charge of the surface, is insufficient to obtain a steady flow, and the electroosmotic transfer in them ceases.

At the same time, the motion of ions in an electric field in such capillaries is preserved and, consequently, electric current $I$ is conserved, if the porosity of the membrane neither changes significantly, nor the current decreases. However, the volume of transferred fluid $V$ will decrease, which should lead to decrease $V / I$. Decrease $V / I$ and $\xi$ should also be proportional to the ratio of the cross-sectional area of large capillaries not participating in electroosmotic transport to the total area of the capillary membrane section. Further improvement of the electroosmotic mass transfer equation leads to the expression 3 for the steady-state regime along the axis parallel to vector $E$ :

$$
\begin{gathered}
\Delta p=\frac{8 \cdot \varepsilon_{a} \cdot(-\xi)}{r_{0}^{2}} \cdot \Delta \varphi-\frac{8 \cdot \eta \cdot l}{S \cdot r_{0}^{2}} \cdot G \\
I=\frac{\sigma \cdot S}{l} \cdot \Delta \varphi,
\end{gathered}
$$

where $\Delta p$ - the electroosmotic pressure, $\varepsilon_{a}$ - dielectric constant of the disperse phase, $\xi$ - current potential, $\Delta \varphi$ the potential difference of the electric field on the membrane, $\eta$ - viscosity of the dispersion medium, $l$ - the length of the membrane; $S$ - the living section of the membrane, $r_{0}$ radius of the cylindrical hole (pore) in the septum (membrane), $G$ - fluid flow through the capillary, $\sigma$ electrical conductivity of the liquid.

Obviously, these dependencies describe real processes in the case of homogeneous dispersions and in the steady-state operating mode.

When working with real heterogeneous systems and taking into account the relaxation phenomena, there are deviations that can be taken into account by introducing corrections for the $\xi$ potential [2].

In the modern era, obviously, the most important criterion of the efficiency of the technological process is energy efficiency - the specific energy inputs for the production. 
Applied to the technology of electrowatering, this can be the specific energy consumption for the displacement of a unit of water volume.

Knowing the water flow and energy consumption for the process, one can calculate the specific energy consumption for water allocation:

$$
w=W / M_{x},[k W \cdot h / k g],
$$

where $W$ - energy costs per process, $k W \cdot h ; M_{x}$ weight of allocated water, $\mathrm{kg}$.

Further, knowing the initial moisture content of material $B_{1}$ and specifying required final $B_{2}$, let us calculate the mass of water that must be extracted from material $M_{x}$, using the known equations of the material balance of the separation process [3].

For the technology of moisture transfer by electroosmosis, provided that the water content in the withdrawn liquid fraction $B_{x} \approx 1$, the following can be obtained:

$$
\begin{aligned}
& M_{1}=M_{x}+M_{2} \\
& M_{1} \cdot\left(1-B_{1}\right)=M_{x} \cdot\left(1-B_{x}\right)+M_{2} \cdot\left(1-B_{2}\right) \\
& M_{1} \cdot B_{1}=M_{x} \cdot B_{x}+M_{2} \cdot B_{2} \\
& M_{x}=M_{1} \cdot \frac{B_{2}-B_{1}}{B_{2}-B_{x}} \\
& \left.\begin{array}{l}
M_{1}=M_{x}+M_{2} \\
M_{1} \cdot\left(1-B_{1}\right)=M_{2} \cdot\left(1-B_{2}\right) \\
M_{1} \cdot B_{1}=M_{x} \cdot B_{x}+M_{2} \cdot B_{2} \\
M_{x}=M_{1} \cdot \frac{B_{2}-B_{1}}{B_{2}-B_{x}}
\end{array}\right\},
\end{aligned}
$$

where $M_{1}, M_{x}, M_{2}$ - the masses of the initial mixture, the liquid fraction withdrawn from the mixture, and the resulting processed particulate material, $\mathrm{kg} ; B_{1}, B_{x}, B_{2}$ - the water content or the dispersed phase in the feed mixture, in the liquid fraction and the resulting particulate material

Next, one can determine the total energy consumption of the process:

$$
W=w \cdot M_{x},[k W \cdot h] .
$$

\section{EXPERIMENT}

Knowing the electrical resistivity of the material and the voltage at the electrodes, it is possible to determine the operating current of the installation and the processing time.

The difficulty lies in the fact that in the electric humidification of the root layer of the soil, it is necessary to take into account the electrical characteristics of each layer of soil and the underlying layers of the soil, in contrast to the water-fall technology [4], where the gradient of the electric field is directed along the layers.

Besides, processes and phenomena accompanying electroosmosis should be taken into account: loss of pressure, loss of moisture conductivity, loss of osmosis, loss of electrolysis, loss of polarization of the dielectric of the dispersed phase, loss of electrical heating of the liquid, operation of thermo-osmosis, diffusion, iontophoresis, dipolar phoresis [5].

The distribution of energy fluxes, characterizing thermodiffusion and electrodiffusion-phosphorous processes, indicates that only part of the energy of electricity will go into losses, while some will participate in useful mass transfer in the form of thermal conductivity, ionosmosis and iontophoresis. Similarly, the distribution of energy flows of other diffusion processes $[6,7]$.

It is known that relaxation processes occur in all processes occurring along with electroosmosis, which determine the values of the relaxation time constants in the corresponding force gradient fields.

The calculation of relaxation phenomena allows a sharp reduction in the specific energy consumption per process $[8$, 9] and the implementation of the electrowatering technology is cost-effective.

The feasibility study of the proposed technology on the example of growing vegetables in the open ground on irrigated lands gave the following prognosis: when irrigation by means of electroosmotic lifting carried out from groundwater, the economic effect will be achieved by reducing labor costs for irrigation and cultivation, fuel consumption by eliminating the work of sprinklers $[10,11]$.

Reduction of capital expenditures for the machine and tractor fleet, reduction of capital investments by eliminating the costs of constructing the irrigation system, increasing the useful area by involving lands occupied by irrigation canals and technological driveways for irrigation equipment. Calculations were carried out for the steppe zone of the Novosibirsk region on the example of growing cabbage.

The basic version for an area of 140 hectares contains irrigation canals and driveways for sprinklers with an area of 5.8 hectares, output of commercial products is 5450 tons, diesel fuel for inter-row processing and irrigation works 54.3 tons, technological equipment in the form of tractors, cultivators and sprinklers, irrigation System in the form of irrigation canals and pumping station. For a season it is required to submit on fields 834000 tons of water at norm of watering $600 \mathrm{~m}^{3} / \mathrm{ha}$.

In the proposed version, the economic effect will be 
ensured by increasing the yield of commercial products from: increasing the production area, increasing phytoproductivity in rainy weather - it is possible to reverse the power supply and create the optimum humidity of the root layer of the soil, reduce the moisture loss by evaporation 4 times.

This will require the construction of an electrical substation with a capacity of about $2000 \mathrm{kV} \cdot A$ and the installation of pulsed power supply formers to 60 $k V \cdot A / h a$; the electricity costs per season will be about $10500000 \mathrm{~kW} \cdot h$.

\section{CONCLUSIONS}

It is established that the optimal physical process for dewatering the slurry can be an electroosmos.

In order to shorten the time of reclamation of slurry barns, it is advisable to remove the relaxation of various power gradient fields by changing the parameters of the electric power supplied to the electrodes.

It is planned to carry out the technological cycle of reclamation in one summer season, during which, with the help of electroosmosis, to lower the slurry moisture and concentration of chemical contaminants to an acceptable level, and then to plant the barn with siderates and thus to return the territory to agricultural circulation.

The proposed technology will make it possible to get rid of a number of irrationally used natural resources.

This is especially true for the Crimean region and the Volga region. Using this technology in Uzbekistan would allow the elimination of the Aral catastrophe and restoration of the natural balance in this region.

\section{References}

[1] E. Y. Abramov, N. I. Schurov and M. V. Rozhkova, "Electric transport traction power supply system with distributed energy sources", IOP Conference Series: Materials Science and Engineering, 2016, Vol. 127, 7 p.

[2] E.Y. Abramov, A.A. Stang and S.A. Enkudinov, "Transformation of the urban electric transport system when using autonomous energy sources", Advanced Materials Research, 2014, Vol. 1040, pp. 778-783.

[3] V.I. Sopov, N.I. Schurov, Y.A. Prokushev and A.A. Shtang, "Increasing the efficiency of the use of electrical energy in the sub-subsystem of electric transport (Povishenie effektivnosti ispol'zovaniya elektricheskoy energii v subpodsisteme elektricheskogo transporta)", Improvement of technical means of electric transport (Sovershenstvovanie tehnicheskih sredstv elektricheskogo transporta), 2002, 189 p. [Digest of scientific works of the NSTU]

[4] B.A. Arzhannikov and A.A. Pyshkin, "Improving of DC power supply system based on automatic voltage regulation of traction substations (Sovershenstvovanie sistemi elektrosnabzheniya postoyannogo toka na osnove avtomaticheskogo regulirovaniya napryazheniya tyagovih podstanciy)", Ekaterinburg: "USURT Press", 2006, 116 p.

[5] V.I.Sopov, V.V. Biryukov, Y.A. Prokushev and Y.A. Rylov, "Analysis of power supply systems for rolling stock with various schemes of traction networks (Analiz sistem elektrosnabzheniya podvizhnogo sostava s razlichnimi shemami tyagovih setey)", Transport, Science, Engineering, Management (Transport, Nauka, Technika, Upravlenie). Vol. 2, 2008, pp. 49-53. [Digest of the VINITI RAS]

[6] V.V. Biryukov and A.V. Kulekina, "The calculation features of the electrical energy storage devices parameters in transport", The 11 International forum on strategic technology (IFOST 2016), pp. 41-43.

[7] N.I. Schurov, E.A. Spiridonov and A.V Larin, "Modes of traction power supply system in case of electric rolling stock equipped with energy storage", Applied Mechanics and Materials, 2014, Vol. 698, pp. 19-23.

[8] Photovoltaic (PV) research: National Renewable Energy Laboratory (NREL). Retrieved on http://www.nrel.gov/ncpv.

[9] N.W.A. Lidula and A.D. Rajapakse, "Microgrids research: a review of experimental microgrids and test systems", Renewable and Sustainable Energy Reviews, 2011, Vol. 15, pp. 186-202.

[10] B. Kroposki, R. Lasseter, T. Ise, S. Morozumi, S. Papathanassiou and N. Hatziargyriou, "A look at microgrid technologies and testing projects from around the world, making microgrids work", IEEE Power and Energy Magazine, 2008, Vol. 6, pp. 40-53.

[11] V.E. Rozenfeld, "Analytical calculation of electric railway networks (Analiticheskiy raschet setey elektricheskih zheleznih dorog)", The theoretical and scientific-practical peer-reviewed journal "Elektrichestvo", 1947, Vol. 9, pp. 6-17. 